\title{
Digital Timing Slack Monitors and Their Specific Insertion Flow for Adaptive Compensation of Variabilities
}

\author{
Bettina Rebaud ${ }^{1,2}$, Marc Belleville ${ }^{1}$, Edith Beigné ${ }^{1}$, Christian Bernard ${ }^{1}$, \\ Michel Robert ${ }^{2}$, Philippe Maurine ${ }^{2}$, and Nadine Azemard ${ }^{2}$ \\ ${ }^{1}$ CEA, LETI, MINATEC, F38054 Grenoble, France \\ \{firstname.name\}@cea.fr \\ ${ }^{2}$ LIRMM-CNRS - Universite Montpellier II, \\ 34392 Montpellier, France \\ \{firstname.name\}@lirmm. fr
}

\begin{abstract}
PVT information is mandatory to control specific knobs to compensate the variability effects. In this paper, we propose a new on-chip monitoring system and its associated integration flow, allowing timing failure anticipation in real-time, observing the timing slack of a pre-defined set of observable flipflops. This system is made of specific structures located nearby the flip-flops, coupled with a detection window generator, embedded within the clock-tree. Validation and performances simulated in a $45 \mathrm{~nm}$ technology demonstrate a scalable, low power and low area, fine-grain system. The integration flow results exhibit the weak impact of the insertion of this monitoring system toward the large benefits of tuning the circuit at its optimum working point.
\end{abstract}

\section{Introduction}

In order to face PVTA (Process Voltage Temperature Aging) variability issues [1-3], and to reduce design margins due to traditional corner-based methodologies, it is possible to reach the optimal operating point of the manufactured chip and thus, get rid of over-pessimism, in using dynamic adaptation of the circuit performances. This solution requires tunable knobs like programmable supply voltages, body biasing or scalable frequencies. In order to efficiently tune those parameters, a monitoring system, providing a real time and accurate diagnostic of the circuit has to be implemented. Two monitoring means have been proposed: (a) integrating specific nonfunctional structures or sensors [4-7]. (Those sensors can be difficult to calibrate, and are only sensitive to global variations) and (b) monitoring directly the sampling elements of the chip (Latches or D-type Flip Flop) [8-12] to detect the occurrence of delay faults. Contrary to previous works, the second solution can detect local variations and is much easier to use due to simple binary output data. On the other hand, to obtain good circuit coverage, many sensors have to be inserted. Solutions [8-10] proposed in line with this approach, i.e. solutions aiming at monitoring the critical paths, have several drawbacks like (a) short paths management imposing buffer insertion, (b) need of a replay or correction systems when an error is detected, (c) high timing 
sensitivity to voltage or frequency scaling and process variations. [12] removes (a-b) disadvantages in suggesting anticipation before error occurrence. However, the propounded implementation does not fit well with frequency or voltage scaling.

Within this context, the contribution of this work is to propose a new monitoring system in line with critical paths monitoring concept, aiming at improving existing implementations and anticipating timing violations over a wide range of operating conditions. The proposed system monitors locally, at run time, on one or more slow paths, critical timing slacks and discloses their evolution with PVT variations or ageing phenomena. One key feature of the system consists in generating a detection window directly using the clock tree architecture to distribute it efficiently to several sensors.

The paper is organized as follows: Section 2 presents the whole monitoring system features, its insertion close to the flip-flops and the specific structures. In section 3, the specific integration flow is detailed, applied with specific constraints. The section 4 provides simulation results of the whole system, validating the monitoring concept proposed.

\section{Monitoring System Proposal}

The proposed monitoring system (Fig.1) is composed of two blocks, designed as standard cell library elements: a sensor (Fig. 3) and a specific Clock-tree Cell (CC) (Fig. 4). The sensor is inserted close to the D-type Flip-Flops (DFF) located at the endpoints of critical timing paths of the design while, the $\mathrm{CC}$ are inserted within the associated clock leafs. Different cells sizing is proposed within the library allowing, if necessary, fine timing tuning.

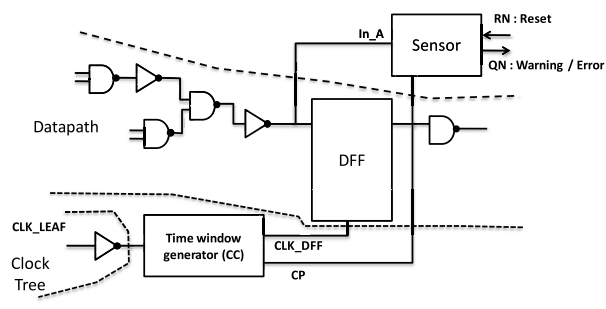

Fig. 1. Proposed monitoring system implemented on a critical path endpoint

As shown Fig.1 and 3, the sensor, acting as a stability checker, is directly connected to a data path output, i.e. to the DFF input. It also receives on one of its input the signal $\mathrm{CP}$ periodically provided by $\mathrm{CC}$, which can drive several sensors. Edges of $\mathrm{CP}$ signal are in phase with those of the clock. The basic function of the sensor is to detect the occurrence of a full or partial transition of the signal In_A during the detection window (of duration Dpulse) positioned in time by the rising edge of $\mathrm{CP}$ as shown Fig. 2. More precisely, this detection window starts at (In-to-CP + CP-toCLK_DFF) before the rising edge of the clock and ends at (In-to-CP + CP-toCLK_DFF - Dpulse) due to some timing characteristics of both sensor and CC. A key 
point here is that (In-to-CP + CP-to-CLK_DFF - Dpulse) must be greater than, or at least equal to, the setup time (Tsetup) of the monitored DFF to detects timing warnings rather than timing errors. In-to-CP is a timing characteristic of the sensor due to its internal inverters [11], and CP-to-CLK_DFF the time interval separating the rising edges of CP and CLK_DFF.

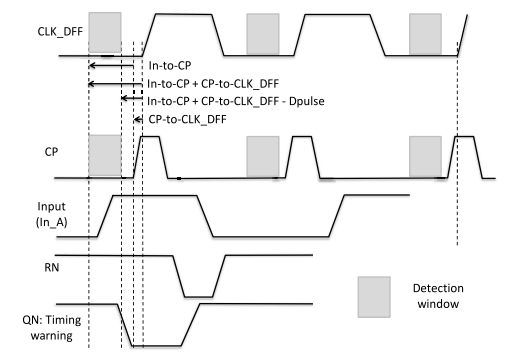

Fig. 2. Transition detection chronogram

If a transition occurs within the detection window, the monitor latches an error signal meaning that, during the last clock cycle, PVT conditions and the data processed by the monitored logic are such that the timing slack (before occurrence of a setup time violation) is lower than Tm $=$ (In-to-CP + CP-to-CLK_DFF - Tsetup $)$. Considering those timing characteristics, sensors are able to warn an imminent system timing failure by detecting the occurrence of a signal transition within the detection window.

To tackle the complexity of actual embedded systems, made of various functional blocks with different levels of timing criticality or working under different operating conditions (Vdd, multiple clock domains), several clock tree cells CC (Fig. 4) generating different time window widths Dpulse and thus, different guard margins Tm must be available in the specific standard cell library.

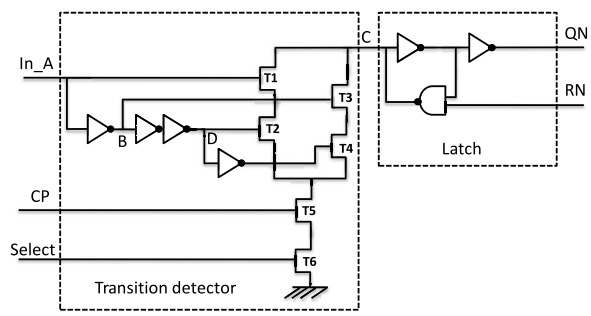

Fig. 3. This one-input sensor works with the load and the discharge of the $\mathrm{C}$ node. In order to reduce the area, several transition detectors can share the same latch. In this condition, $\mathrm{C}$ is bigger and the structure slower: trade-off has to be made.

Performance and system validation results obtained considering a $45 \mathrm{~nm}$ Low Power technology are given in [11]. Those results demonstrate that the implemented structures are robust to power supply, frequency and temperature variations. More precisely, it is shown in [11] that: 
- the 4-input sensor can detect the occurrence of transitions until 0.6V @ 125C in worst case process, and has low process induced delay variations.

- the clock cell CC provides reliable detection window(s) whatever the environmental conditions are.

- the system can achieve interesting timing margin reduction (w.r.t. to worst case timing conditions).

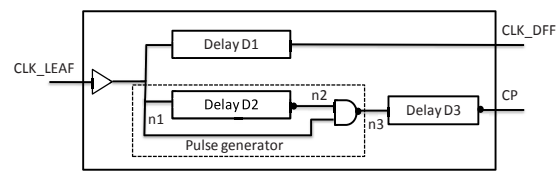

Fig. 4. Example of specific clock cell (CC) implementation

Sensor cells with 1 and up to 4 inputs and a programmable CC with W1 and W2 detection windows have been designed, layout drawn and characterized in $45 \mathrm{~nm}$ Low Power technology, in order to create a new standard cell library. Sensors were characterized as DFF since their timing behavior is quite similar to that of DFF. The description of CC was done accordingly to the description of any clock buffer.

\section{Integration Flow}

In order to demonstrate the whole system efficiency, in terms of functionality and integration easiness, a test chip has been designed, and a dedicated digital flow has been studied (Fig. 5). Further details on test chip are given section 4. This section aims at describing this specific flow, and the new timing constraints that must be taken into account for a generic block.

As shown Fig.5, three steps are required to integrate the proposed monitoring system. In a first step, a prototype (a placed and routed design) is obtained in order to identify the critical paths to be monitored, and to get an accurate description of the

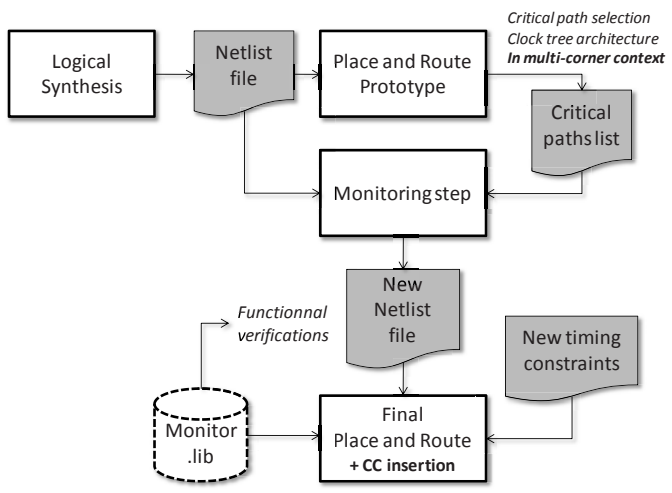

Fig. 5. Flow description to integrate the proposed monitoring system 
clock tree. This step achieved, sensors and dummy cells are inserted in the netlist. Then, starting from the prototype results obtained from step 1, final place and route steps are performed, considering additional timing constraints related to $\mathrm{CC}$ and sensors insertion. In this last step, balancing the nets between DFF and their associated sensor is a key design guideline.

\subsection{Critical Paths Choice}

Getting an exhaustive coverage of our test chip is unrealistic as the numbers of sensors will double the occupied area and the power consumed by the sequential elements. Thus, some critical paths to be monitored have to be selected.

One possible solution is to use Statistical Static Timing Analysis [13-14]. SSTA is performed to identify the paths (assuming that these paths are correlated enough) with the highest probabilities of violating the setup time constraint. However, with the decreasing transistor length and the increasing impact of local and random variations, correlations between timing paths shrink, leading to a growing number of paths that have to be monitored to remain sure to detect warning signal before a failure.

Therefore, another selection policy was adopted since our goal was to prevent a system failure before the occurrence of any timing violation (no timing error is allowed in the system). Considering this constraint, our strategy is to impose specific target slack constraints during the synthesis and the place and route steps. More precisely, specific target slack constraints were chosen to create the set of pre-defined critical paths to be monitored. Specifically, we intentionally relax the target slack constraint of a set of critical paths. This set includes the worst critical path which has been identified during synthesis runs at worst case and tight timing constraints.

This results in a timing slack distribution characterized by two distinguishable sets of paths (illustration Table 1). A reduced set of paths characterized by a reduced timing slack forms the set of paths to be monitored, and a huge set of paths characterized by larger timing slacks, and more precisely by slacks 100 ps greater than the most critical path (which is in the first set of paths).

Table 1. Number of critical path endpoints with a delay value lower than that of the worst critical path by less than $100 \mathrm{ps}$ (synthesis step at worst timing corner $1,05 \mathrm{~V}, 125^{\circ} \mathrm{C}$ )

\begin{tabular}{lll}
\hline & $\begin{array}{l}\text { \# critical paths } \\
\text { In 100 ps from the WNS }\end{array}$ & $\begin{array}{l}\text { Power }(\mathrm{mW}) / \\
\text { Max Freq }(\mathrm{ns})\end{array}$ \\
\hline Typical flow & 3695 & $29 / 1,6$ \\
\hline New flow & 24 & $35 / 1,7$ \\
\hline
\end{tabular}

Such timing slack distribution is obtained thanks either to multi-mode capabilities of CAD tools or to specific relaxing tool commands. However, it is of prime importance to warrant that the paths to be monitored are representative of the circuit operating in any possible conditions. Thus a verification step is required in order to fulfill this condition, using either multi-corner or statistical simulations. This specific flow results in over-constraining some paths from a timing point of view that consists mainly in increasing the power consumption as shown Tab.1. However, the overhead is expected to be significantly lower than the gains achieved by applying adaptive voltage, body-biasing and frequency scaling techniques. 


\subsection{Monitoring Step and Specific Place and Route Constraints}

The monitoring phase is performed after the true critical paths identification, in all operating conditions, before the final place and route step. As shown Fig.5, the monitoring phase aims at providing: (a) a new netlist file including dummy cells and sensors, as shown Fig. 6, and (b) a new timing constraints file to guide the place and route step. This step can be fully automated by developing specific scripts to ease the monitor integration. It is also possible to integrate some sensors such as they share several flip-flops; however this is highly dependent on the clock tree structure. As a result, 4 DFF sharing the same sensor has been found as a practical limit in our testcase.

Moreover, Clock Cells (CC) cannot be inserted during the monitoring phase since it would induce some setup and hold timing violations during functional verifications in locally modifying the clock skew. Furthermore, this specific point is highly critical if clock gating is applied to reduce the dynamic power consumption. As a result, the clock cell insertion is pushed to the next step.

(a)

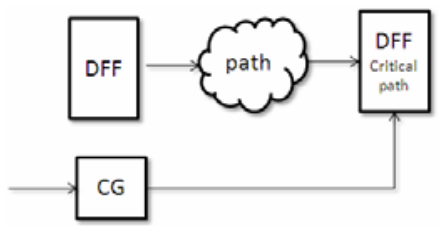

(b)

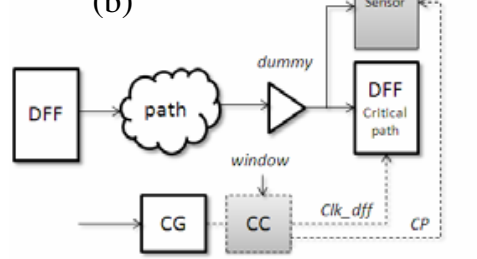

Fig. 6. Critical path before (a) and after (b) the monitoring step. Sensors are added with specific dummy cells allowing an easy place and route. Clock Cells are inserted later.

To ensure that no skew will appear between the event going to the sensor and the same one going to the $\operatorname{DFF}(\mathrm{s})$, and thus keep the Tm margin safe, specific timing constraints must fixed in the constraint file considered during the final place and route step. This timing specifications aim at constraining the timing driven place and route tool such as the timing fork between the $\operatorname{DFF}(s)$ and the related sensor remains well balanced. This can be done by imposing a specific timing constraint to the dummy cell driving the sensor and its associated DFF. Furthermore, additional hold time issues introduced by the $\mathrm{CC}$ detection window (when the window is still open after the rising clock edge) have also to be considered and fixed by adding some specific constraints in the timing constraint file. However, the reduced detection window width (compared to [8-10]) enables to insert a limited number of buffers in short paths.

\subsection{Specific Clock Tree Cell Insertion}

As the clock tree architecture may be highly sensitive to PVT variations, CC are inserted as close as possible to the monitoring path endpoint (Fig. 7) thanks to the additional timing constraints introduced at the monitoring phase. This method leads to 
- reduce the impact of spatial correlations on the timings of the different monitoring elements.

- limit the impact of local voltage drops and temperature gradients on timings.

- reduce the impact on timings of interconnects variability.

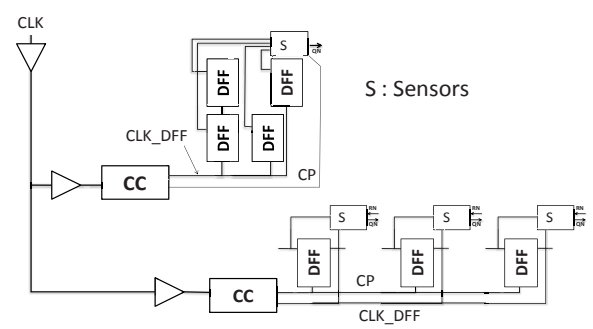

Fig. 7. Final clock tree architecture example with two CC driving 7 DFF and 4 sensors

As a result, Clock Cell insertion is performed at the Place and Route level, after a first clock tree synthesis (CTS). The insertion done, a second CTS is run in order to balance the tree, and minimize the global skew of the design before the final routing.

\section{Results}

This section describes the main results while using the dedicated flow (Fig. 5), by pointing out the new constraints efficiency and the validation of the monitoring system concept.

The monitoring system has been integrated in an arithmetic and reconfigurable block incorporating: 4 SRAMs, several register banks, and computing elements such as dividers or Multipliers-Accumulators (MAC). This block, inserted in a telecom SoC was considered as an ideal test case because of its timing criticality. Due to the complexity of the considered real time application, no replay of instruction sets was feasible at system level, and timing performances have to be high enough to satisfy a strained real time environment. Designed in $45 \mathrm{~nm}$ STMicroelectronics Low Power technology, it contains about 13400 flip-flops, which leads to a $606 * 558 \mu \mathrm{m}^{2}$ core floorplan implementation. The timing constraint period is $1.5 \mathrm{~ns}$ with nominal process libraries, @ $25^{\circ} \mathrm{C}$ and $1,1 \mathrm{~V}$.

Critical path endpoint selection. Applying the specific flow on the placed and routed prototype, a set of 160 critical path endpoints, defining a set of critical paths, situated at the output of the first stage of the pipelined MAC, was isolated. The maximum operating frequency Fmax was characterized in nominal conditions. Values of $1.78 \mathrm{~ns}$ and $1.36 \mathrm{~ns}$ were estimated as the minimal periods allowing correct timing behaviors of respectively the typical and best endpoints among this set of critical path endpoints.

To reduce the number of endpoints to be monitored we ran a multi-corner analysis to refine our initial selection since the worst endpoint may differ from one PVT conditions to another. To select this reduced set of endpoints to be monitored, we analyzed successively the timing behaviors of the worst, the 10 worst and finally the 50 worst endpoints in nominal conditions (nom 1,1V 25C). Tab 2 gives the obtained simulation results. 
The worst endpoint obtained in nominal conditions remains the worst one in most PVT conditions except when the worst case process and voltage corner are considered. Thus monitoring only this endpoint is proved to be deficient to cover the timing behavior of the whole circuit. Considering 10 worst endpoints in nominal corner rather than the worst one improves the results since at least 7 (i.e. $70 \%$ ) of these endpoints remain among the 10 critical endpoints in all other PVT conditions. We therefore increased the number of considered critical endpoints successfully to 50 . The results obtained were similar to the results obtained considering 10 worst endpoints: none of these subsets of endpoints are always the most critical in all PVT conditions.

Table 2. Selecting a reduced set of critical endpoints

\begin{tabular}{|c|c|c|c|c|c|c|}
\hline & \multicolumn{6}{|c|}{$\begin{array}{c}\text { Number (and percentage (\%)) of worst endpoints obtained in nominal } \\
\text { conditions remaining among the n worst in other PVT conditions } \\
\text { Worst endpoint captured (yes or no) }\end{array}$} \\
\hline & $\begin{array}{c}\text { bc } 1,2 \mathrm{~V} \\
\mathrm{~m} 40 \mathrm{C}\end{array}$ & $\begin{array}{c}\text { bc } 1,2 \mathrm{~V} \\
125 \mathrm{C}\end{array}$ & $\begin{array}{c}\text { nom } 1,1 \mathrm{~V} \\
25 \mathrm{C}\end{array}$ & $\begin{array}{c}\text { wc } 1,05 \mathrm{~V} \\
125 \mathrm{C} \\
\end{array}$ & $\begin{array}{c}\text { wc } 0,9 \mathrm{~V} \\
125 \mathrm{C}\end{array}$ & $\begin{array}{c}\text { we } 0,9 \mathrm{~V} \\
\text { m40C }\end{array}$ \\
\hline The worst endpoint $(n=1)$ & 1 (100) & 1 (100) & 1 (100) & 1 (100) & $0(0)$ & $0(0)$ \\
\hline The 10 worst endpoints & $8(80)$ & $8(80)$ & $10(100)$ & $8(80)$ & $7(70)$ & $7(70)$ \\
\hline$(n=10)$ & yes & yes & yes & yes & yes & Yes \\
\hline The 25 worst endpoints & $22(88)$ & $21(84)$ & $25(100)$ & $23(92)$ & $22(88)$ & $22(88)$ \\
\hline$(n=25)$ & yes & yes & yes & yes & yes & Yes \\
\hline The 50 worst endpoints & $46(92)$ & $45(90)$ & $50(100)$ & $45(90)$ & $45(88)$ & $45(88)$ \\
\hline$(\mathbf{n}=\mathbf{5 0})$ & yes & yes & yes & yes & yes & Yes \\
\hline max frequency $(\mathrm{Mhz})$ & 763 & 735 & 561 & 422 & 270 & 212 \\
\hline min period (ns) & 1,31 & 1,36 & 1,78 & 2,37 & 3,7 & 4,7 \\
\hline $\begin{array}{l}\text { Greatest Negative Slack } \\
\text { obtained considering the } 10 \\
\text { worst endpoints (ps) }\end{array}$ & 56 & 68 & 60 & 100 & 150 & 190 \\
\hline $\begin{array}{l}\text { Greatest Negative Slack } \\
\text { obtained considering the } 50 \\
\text { worst endpoints (ps) }\end{array}$ & 98 & 106 & 164 & 219 & 205 & 297 \\
\hline
\end{tabular}

However, we noticed that the worst endpoint at each considered PVT conditions was always in the sets of 10 and 50 worst endpoints obtained in nominal condition. We thus conclude that monitoring these 10 or 50 worst endpoints instead of 160 was a good warranty to monitor the worst endpoint for all considered PVT conditions (in Tab 2 a 'yes' means that the worst endpoint at the considered PVT conditions is in the $\mathrm{n}$ worst endpoint set defined in nominal conditions). We decided to monitor the 50 worst endpoints to cover a larger window of arrival times; as shown Tab 2 the greatest negative slack considering 50 endpoints is roughly 1.3 to 2.7 times larger than the slack obtained considering only 10 paths.

Clock tree architecture, monitoring and CC insertion. Analyzing the clock tree architecture is mandatory to know which standard CC cells have to be used and where they will be inserted. The first clock tree synthesis applied in our specific flow leads to a 15 level clock tree, with 1404 sub trees, monitoring 13400 flip-flops through 530 clock gating elements. The clock tree latency was about $1.19 \mathrm{~ns}$ in nominal corner condition, with a skew of 97 ps. These values are given at the end of the place and 
route prototype. Considering the endpoints to be monitored, the number of inserted sensors in our design was 19 (to monitor the 50 endpoints), with $11 \mathrm{CC}$ (timing pulse $150 \mathrm{ps}$ in nominal corner), with the design policy that close path endpoints are gathered on a same sensor with a limit of 4 paths by sensors, and that each clock cell must be inserted at the clock leaf. After the monitoring step and the second place and route phase (with two clock tree synthesis and new timing constraints), the final clock skew obtained in nominal case was 102 ps with a latency of 1.28 ns. Performing a two back-end run flow in identifying the critical paths during the first run did not imply enough classification changes which can modify the efficiency of the selected set. As a result, we may conclude that the whole monitoring flow is functional and efficient, since (a) the skew has not increased much, (b) the sensors and their related endpoint flip-flops are not physically distant of more than $4 \mu \mathrm{m}$ (i.e. about 2 standard cell height) and (c) the critical path set is still applicable.

Table 3. FWi/Fmax ratio for a typical process corner

\begin{tabular}{ccccccccc}
\hline & W1 ratio & \multicolumn{7}{c}{ W2 ratio } \\
\hline Temp/Power & $\mathbf{0 , 9}$ & $\mathbf{1}$ & $\mathbf{1 , 1}$ & $\mathbf{1 , 2}$ & $\mathbf{0 , 9}$ & $\mathbf{1}$ & $\mathbf{1 , 1}$ & $\mathbf{1 , 2}$ \\
\hline $\mathbf{- 4 0}$ & 95 & 93.6 & 93.9 & 92.4 & 92.1 & 91,2 & 90,6 & 89,7 \\
$\mathbf{2 5}$ & 92.7 & 92.5 & 92.7 & 92.5 & 89.8 & 89,2 & 89,4 & 89,9 \\
$\mathbf{7 5}$ & 91.8 & 92.3 & 90.5 & 89.8 & 89.3 & 89 & 87,4 & 87,3 \\
$\mathbf{1 2 5}$ & 92.1 & 90.5 & 90.3 & 90 & 88.8 & 87,9 & 87,2 & 87,5 \\
\hline
\end{tabular}

Behavior validation and performances. In order to check if the whole system remains functional, we extracted from simulation (a) the maximum operating frequency Fmax of the circuit at different PVT conditions (b) the maximum frequencies FW1 and FW2 at which the monitoring system does not flag any timing warnings or violations, considering respectively two different detection windows $\mathrm{W} 1$ and $\mathrm{W} 2$; W2 corresponding to the largest detection window. Table 2 gives the simulated results for FW1/Fmax and FW2/Fmax ratios at different VT conditions for a nominal process (other process corners demonstrate similar efficiency):

- FW1/Fmax and FW2/Fmax ratios remain lower than 100\% meaning that the monitoring system operates correctly and warns an imminent timing failure, in every PVT conditions.

- Considering the global PVT conditions, the timing margins Tm(W1) and $\mathrm{Tm}(\mathrm{W} 2)$ remain respectively between $80 / 340$ and $120 / 480$ ps when the system operates correctly.

These results demonstrate the efficiency of the monitoring system in making it particularly interesting for adaptation: the circuit can work at roughly $90 \%$ of its maximal speed under all PVT conditions and the system is very attractive in nominal and best cases to crop margins or decrease consumption. It can also be favorable to integrate two different detection windows to control the timing margins with accuracy during the voltage scaling. 


\section{Conclusion}

This paper describes a new in situ monitoring system, based on the insertion of sensors close to observable flip-flop endpoints, coupled with specific clock tree cells providing detection windows. This system prevents from timing violations rather than detecting an error. Valuable and simulated in a wide range of environmental conditions, this timing slack monitoring system, compact and with little impact on the overall power consumption, can thus be used together with knob-based adaptive solutions.

A dedicated flow based on a standard design flow was described, easing the insertion of the specific detection material and allowing a clear critical path endpoint choice to reduce the number of paths to be monitored. Simulation results demonstrate the efficiency of such a system and its easy integration, making it very attractive to work at the optimal PVT operating point for critical IP blocks in advanced technologies.

\section{References}

1. Narayanan, V., et al.: Proc. 18th ACM Great Lakes Symposium on VLSI, Orlando, Florida, USA (2008)

2. Lasbouygues, B., et al.: Temperature- and Voltage-Aware Timing Analysis. IEEE Trans. on CAD of Integrated Circuits and Systems 26(4), 801-815 (2007)

3. Parthasarathy, C., Bravaix, A., Guérin, C., Denais, M., Huard, V.: Design-In Reliability for 90-65nm CMOS Nodes Submitted to Hot-Carriers and NBTI Degradation. In: Azémard, N., Svensson, L. (eds.) PATMOS 2007. LNCS, vol. 4644, pp. 191-200. Springer, Heidelberg (2007)

4. Nourani, M., Radhakrishnan, A.: Testing On-Die Process Variation in Nanometer VLSI. IEEE Design \& Test of Computers 23(6), 438-451 (2006)

5. Samaan, S.B.: Parameter Variation Probing Technique: US Patent 6535013 (2003)

6. Persun, M.: Method and apparatus for measuring relative, within-die leakage current and/or providing a temperature variation profile using a leakage inverter and ring oscillators: US Patent 7193427 (2007)

7. Drake, A., et al.: A Distributed Critical Path Timing Monitor for A 65nm High Performance Microprocessor. In: ISSCC, pp. 398-399 (2007)

8. Das, S., et al.: A Self-Tuning DVS Processor Using Delay-Error Detection and Correction. IEEE JSSC 41(4), 792-804 (2006)

9. Blaauw, D., et al.: Razor II: In situ error detection and correction for PVT and SER tolerance. In: ISSCC, pp. 400-401 (2008)

10. Bowman, K.A., et al.: Energy-Efficient and Metastability-Immune Timing-Error Detection and Instruction-Replay-Based Recovery Circuits for Dynamic-Variation Tolerance. In: ISSCC, pp. 402-623 (2008)

11. Rebaud, B., et al.: An Innovative Timing Slack Monitor for Variation Tolerant Circuits. In: ICICDT (2009)

12. Agarwal, M., et al.: Circuit Failure Prediction and Its Application to Transistor Aging. In: Proc. VLSI Test Symposium, pp. 277-286 (2007)

13. Migairou, V., Wilson, R., Engels, S., Wu, Z., Azemard, N., Maurine, P.: A Simple Statistical Timing Analysis Flow and Its Application to Timing Margin Evaluation. In: Azémard, N., Svensson, L. (eds.) PATMOS 2007. LNCS, vol. 4644, pp. 138-147. Springer, Heidelberg (2007)

14. Blaauw, D., et al.: Statistical timing analysis: From basic principles to state of the art. IEEE Trans. on CAD of Integrated Circuits and Systems 27(4), 589-607 (2008) 